\title{
Barriers and facilitators to the integration of mental health services into primary health care: a systematic review protocol
}

Edith K. Wakida ${ }^{*^{*}}$ (D), Dickens Akena ${ }^{2,3}$, Elialilia S. Okello ${ }^{2,8}$, Alison Kinengyere ${ }^{3,4}$, Ronald Kamoga ${ }^{5}$, Arnold Mindra ${ }^{1}$, Celestino Obua ${ }^{6}$ and Zohray M. Talib

\begin{abstract}
Background: Mental health is an integral part of health and well-being and yet health systems have not adequately responded to the burden of mental disorders. Integrating mental health services into primary health care (PHC) is the most viable way of closing the treatment gap and ensuring that people get the mental health care they need. PHC was formally adapted by the World Health Organization (WHO), and they have since invested enormous amounts of resources across the globe to ensure that integration of mental health services into PHC works.

Methods: This review will use the SPIDER (Sample, Phenomenon of Interest, Design, Evaluation, Research type) framework approach to identify experiences of mental health integration into PHC; the findings will be reported using the "Best fit" framework synthesis. PubMed, EMBASE, PsycINFO, and Cochrane Central Register of Controlled trials (CENTRAL) will be searched including other sources like the WHO website and OpenGrey database. Assessment of bias and quality will be done at study level using two separate tools to check for the quality of evidence presented. Data synthesis will take on two synergistic approaches (qualitative and quantitative studies). Synthesizing evidence from countries across the globe will provide useful insights into the experiences of integrating mental health services into PHC and how the barriers and challenges have been handled. The findings will be useful to a wide array of stakeholders involved in the implementation of the mental health integration into PHC.

Discussion: The SPIDER framework has been chosen for this review because of its suitable application to qualitative and mixed methods research and will be used as a guide when selecting articles for inclusion. Data extracted will be synthesized using the "Best fit" framework because it has been used before and proved its suitability in producing new conceptual models for explaining decision-making and possible behaviors. Synthesizing evidence from countries across the globe will provide useful insights into the experiences of integrating mental health services into PHC and how the barriers and challenges have been handled.
\end{abstract}

Systematic review registration: PROSPERO CRD42016052000

Keywords: Integration, Mental health services, Primary health care, Barriers and facilitators

\footnotetext{
* Correspondence: ediwakida@must.ac.ug

${ }^{1}$ Office of Research Administration, Mbarara University of Science and

Technology, Mbarara, Uganda

Full list of author information is available at the end of the article
} 


\section{Background}

\section{Description of the condition}

Mental health as defined by the World Health Organization is a state of well-being in which every individual realizes his or her own potential, can cope with the normal stresses of life, can work productively and fruitfully, and is able to make a contribution to her or his community [1]. It is an integral part of health and well-being and yet health systems are yet to adequately respond to the burden of mental health problems. Up to $85 \%$ of people with severe mental disorders in low-income and middle-income countries receive no treatment for their disorder [2, 3]. Mental and behavioral disorders are estimated to account for $14 \%$ of the global burden of disease with $19 \%$ of the burden in subSaharan Africa (SSA) [4]. If untreated, mental and behavioral disorders are likely to cause severe disability and heavy socio-economic burden on families and communities [5-9]. Integrating mental healthcare services into primary health care $(\mathrm{PHC})$ is among the most viable means of closing the treatment gap and ensuring that people get the mental healthcare they need $[7,9]$.

\section{Description of the intervention}

$\mathrm{PHC}$ is the first point of contact an individual has with the health system and is essential to making health care universally accessible to individuals and families in the community in an acceptable and affordable way with full participation [10, 11]. It was formally adopted by the WHO through the Alma-Ata declaration as the best method for providing a comprehensive, universal, equitable and affordable healthcare service and that it had the ability to reduce stigma, improve access to care, reduce chronicity of mental illness and improve social integration $[4,12,13]$. The Alma-Ata model of mental health integration recommends that countries build or transform their mental health services to (i) promote selfcare, (ii) build informal community care services, (iii) build community mental health services, (iv) develop mental health services in general hospitals, and (v) limit reliance on psychiatric hospitals [14].

Furthermore, evidence suggests that mental health care can be delivered effectively in PHC settings, and that once identified, most mental illnesses can be treated using costeffective means $[9,15,16]$. Treatment of common mental disorders at $\mathrm{PHC}$ can be improved through collaborative care interventions, yielding better access to care, better physical as well as mental health outcomes, and improved overall cost-effectiveness $[17,18]$.

\section{How the intervention might work}

The WHO issued key recommendations to guide the process of integrating mental health services into PHC; these include (i) doing a preliminary situational analysis of the best options for the treatment and care of mental disorders at different levels of care, (ii) building on existing networks/structures and human resources to provide mental health services, (iii) redistributing funding from tertiary to secondary and primary levels of care or making new funds available, (iv) clear delineation of mental disorders to be treated at the primary care level, (v) training of primary care staff in identification and treatment of mental disorders (vi) recruitment/education of new PHC staff, (vii) availing of basic psychotropic medicines at primary and secondary care levels, and (viii) adequate supervision and support of PHC staff by mental health specialists if integration is to succeed [4].

\section{Why it is important to do this review}

There is a need to strengthen health systems to address the existing treatment gaps for mental health given that the WHO has invested enormous amounts of resources to ensure that integration of mental healthcare services into PHC is achieved [19].

Furthermore, health systems have both the hardware (human resources, finance, medicines and technology, service infrastructure, and information system) as well as the software (ideas and interests, relationships and power, values and norms) [20] at whose center are people. Without putting people first, there are bound to be challenges of integrated healthcare because no established direct relationship between individuals, families in the community, and a specific staff member have been created [7].

Integration of mental health into $\mathrm{PHC}$ has been done by various countries and in different forms [21-24] including training PHC workers to identify mental health problems, assessing for mental illnesses during medical standard of care, PHC providers/community health workers and health care managers working together to address mental health related illnesses, and availing psychotropic medications to PHC centers [25]. In addition, researchers have documented information about barriers and facilitators to the integration process [26-28]. Integration of mental health services, for the purpose of this review, will be defined as blending mental healthcare services (identification, treatment, and or referral) into the medical standard of care. PHC will be defined as the first point of contact with the health system [29].

The review will be based on the SURE (Supporting the Use of Research Evidence) guide that focuses on barriers to implementing health system changes [30, 31] (see Table 1). The SURE framework is among the most robust with regards to the identification of and addressing barriers to implementing policy options; however, it was developed for implementing health changes within Africa. This review will in part validate the SURE guide for global use. It will synthesize and document the barriers and facilitators to the integration of mental health services into $\mathrm{PHC}$ and explain the implementation of 
Table 1 SURE framework for identifying factors affecting implementation of a policy

\begin{tabular}{|c|c|}
\hline Level & Factors affecting implementation \\
\hline \multirow[t]{3}{*}{ Recipients of care } & Knowledge and skills \\
\hline & $\begin{array}{l}\text { Attitudes regarding program } \\
\text { acceptability, appropriateness and } \\
\text { credibility }\end{array}$ \\
\hline & $\begin{array}{l}\text { Motivation to change or adopt } \\
\text { new behavior }\end{array}$ \\
\hline \multirow[t]{3}{*}{ Providers of care } & Knowledge and skills \\
\hline & $\begin{array}{l}\text { Attitudes regarding program } \\
\text { acceptability, appropriateness and } \\
\text { credibility }\end{array}$ \\
\hline & $\begin{array}{l}\text { Motivation to change or adopt } \\
\text { new behavior }\end{array}$ \\
\hline \multirow{3}{*}{$\begin{array}{l}\text { Other stakeholders (including other } \\
\text { healthcare providers, community } \\
\text { health committees, community } \\
\text { leaders, program managers, donors, } \\
\text { policy makers and opinion leaders) }\end{array}$} & Knowledge and skills \\
\hline & $\begin{array}{l}\text { Attitudes regarding program } \\
\text { acceptability, appropriateness and } \\
\text { credibility }\end{array}$ \\
\hline & $\begin{array}{l}\text { Motivation to change or adopt } \\
\text { new behavior }\end{array}$ \\
\hline \multirow[t]{17}{*}{ Health system constraints } & Accessibility of care \\
\hline & Financial resources \\
\hline & Human resources \\
\hline & Educational system \\
\hline & Clinical supervision \\
\hline & Internal communication \\
\hline & External communication \\
\hline & Allocation of authority \\
\hline & Accountability \\
\hline & Management and or leadership \\
\hline & Information systems \\
\hline & Facilities \\
\hline & Patient flow processes \\
\hline & $\begin{array}{l}\text { Procurement and distribution } \\
\text { systems }\end{array}$ \\
\hline & Incentives \\
\hline & Bureaucracy \\
\hline & $\begin{array}{l}\text { Relationship with norms and } \\
\text { standards }\end{array}$ \\
\hline \multirow[t]{8}{*}{ Social and political constraints } & Ideology \\
\hline & Short-term thinking \\
\hline & Contracts \\
\hline & Legislation or regulations \\
\hline & Donor policies \\
\hline & Influential people \\
\hline & Corruption \\
\hline & Political stability \\
\hline
\end{tabular}

Adopted from "The SURE Collaboration, 2011" World Health Organization mental health integration into PHC. The results will be useful to key stakeholders involved in the implementation of the mental health integration into PHC.

\section{Review objectives}

The objectives of this review are to synthesize and document the barriers and facilitators to the integration of mental healthcare services into primary health care.

\section{Methods/design}

The systematic review is registered in PROSPERO international prospective register of systematic reviews (CRD42016052000). It has been written according to the Preferred Reporting Items for Systematic Reviews and Meta-Analyses Protocols (PRISMA-P) recommended for systematic reviews [32]. The checklist is included as an additional file (see Additional file 1). This review will use the SPIDER (Sample, Phenomenon of Interest, Design, Evaluation, Research type) framework approach [33] and the findings will be reported using the "Best fit" framework synthesis [34].

\section{Sample}

The sample or population of interest is health care providers, community health workers, health care managers, and policy makers who have been involved in the integration of mental health into PHC. The listed stakeholders should be involved in general health care, collaborative care, and/or specialized health care. We will include studies from any country in the world involved in the integration of mental health services into PHC.

\section{Phenomenon of interest}

We will include studies that document the integration of mental health services into general healthcare; are delivered at primary or community health care settings; and are collaborative in nature (the primary health care providers, community health workers, and health care managers, working together) so as to understand the how and why of certain behaviors, decisions, and individual experiences of integration of mental health into PHC.

\section{Design}

The theoretical framework in the studies will be used to determine the research method used, while the details of the study design will help to make decisions about the robustness of the study and analysis. In addition, this might increase the detection of qualitative studies in the databases in which titles and abstracts are unstructured [33].

\section{Evaluation}

Evaluation of the outcomes will be done depending on the research question which may include unobservable and subjective constructs like attitudes and views when 
compared to quantitative research [33].The outcomes will be looked at in relation to the SURE Framework as detailed in Table 1 to evaluate the policy option.

\section{Research type}

We will review studies that have documented experiences and attitudes of stakeholders about integration of mental health into general PHC; studies conducted in specialized clinics (HIV, maternal and child health, and reproductive health) will be included. Our search will cover three research types: (i) qualitative studies that used appropriate methods of data collection and analysis (such as ethnography, grounded theory, phenomenology, and case studies) [31, 35, 36]; (ii) quantitative studies; and (iii) mixed methods studies combing qualitative and quantitative methods of data collection and analysis which will include cross-sectional studies, case-control studies, cohort studies, quasi-experimental studies, and randomized control trials.

\section{Inclusion criteria}

To be eligible for inclusion, the articles will be required to describe mental health integration (policy and, or service provision) in PHC settings. They should involve one or more type of care provider (doctor, nurse, social worker, clinical officer, community health worker); deal with any type of mental health (depression, schizophrenia, anxiety, or general mental health) and any age group or population that receive mental health services; and present barriers/challenges, facilitators/enablers of mental health integration into PHC. Articles with aspects of collaborative engagements (primary care providers with mental health specialists and other professionals) will be eligible for inclusion. See Table 2 for categorization of inclusion criteria by SPIDER framework.

Articles not in a PHC or community setting, offering training in mental health integration, and conducting a situation analysis for integration of mental health into PHC will not be eligible for inclusion. The quality of reporting of studies will be considered when selecting articles for inclusion, and inadequately reported studies will be excluded [37].

\section{Search methods for identification of studies}

We will search the following databases to identify potentially eligible studies for review: PubMed, EMBASE, PsycINFO, and Cochrane Central Register of Controlled trials (CENTRAL). The search will be open to any language, no date restriction will be placed, and the search terms will be kept broad to capture potentially eligible studies. An example of the proposed search strategy of PubMed is attached as Additional file 2. In addition, other sources including the WHO website, OpenGrey database, and reference lists from relevant studies reviewed will be searched. Where necessary, experts or specialist authors in the field will be contacted.

\section{Data extraction}

Two review authors (EW and RK) will independently screen the titles and/or abstracts of the identified records for eligibility. The full text of all the papers identified as potentially relevant will then be retrieved by AK and DA. Eligible citations will be read in full-text version by EW, $\mathrm{EO}$, and ZT and evaluated for inclusion using the eligibility criteria. Disagreements will be resolved by consensus and discussion with a third reviewer (CO) when needed. Following the literature search, an EndNote database (EndNote version X7.7.1 Thomson Reuteurs) will be used to manage search results.

Three review authors (EW, AM, and RK) will independently extract data using a pre-tested data extraction form; discrepancies will be identified and resolved through discussion (with the senior researcher on the team $\mathrm{CO}$ where necessary). Where appropriate, we (EW) will contact the corresponding authors of the included studies for clarification and missing data.

Extracted data will include study title, name of the first author, year of publication, country of study, study setting, facility type (public/free at point of use, insurance based, private/nominal payment, NGO, etc.), study type (qualitative, quantitative and mixed methods studies), study population/cadre (health care providers/community health workers,/health care managers including doctors, nurses, clinical officers, social workers among other health cadres and policy makers), and barriers and facilitators (categorized by study type).

The key information will be identified through fulltext review and extracted by study type (qualitative/ quantitative barriers and facilitators separately), after which it will be categorized along the parameters in the SURE framework [30] as detailed in Table 1 in preparation for synthesis.

This review derives its elements from three sources: (1) key information required (population, setting, and

Table 2 Inclusion criteria

\begin{tabular}{ll}
\hline & Primary research studies \\
\hline Setting/population & Primary health care/community \\
Phenomenon of interest & Mental health integration (policy, and/or service provision) \\
Design, evaluation, and research & $\begin{array}{l}\text { Interviews, focus group discussions, surveys (that explored primary care providers' } \\
\text { experiences (barriers and challenges/facilitators and enablers), perceptions, attitudes }\end{array}$ \\
\hline
\end{tabular}


intervention for synthesis and interpretation, (2) SURE framework to guide the implementation of health systems changes, and (3) the quality assessment criteria. Two reviewers will conduct independent quality assessments of the included studies [37] so as to inform judgment of not only the internal validity of included studies but also the validity of the findings of the synthesis [34].

\section{Risk of bias and quality assessment}

Assessment of bias and quality will be done at study level using two separate tools to check for the evidence presented. For qualitative studies, the Critical Appraisal Skills Program (CASP) qualitative checklist [38] will be used while the Effective Public Health Practice Project Quality Assessment Tool will be used for Quantitative Studies [39]. In order to limit publication bias, articles from both published and unpublished data sources will be included, information will be compared to ensure that it is representative of completed studies conducted in the same population, studies published in any language will be included, and there will be no selective reporting of some outcomes to the exclusion of others. This process will be ensured by the primary reviewer (EW) in consultation with EO and ZT.

\section{Primary outcomes}

The primary outcomes of the review will be the barriers (challenges) and facilitators (enablers) to the integration of mental health services into primary health care. We have chosen to use the terms "barriers and facilitators" to describe all factors that might inhibit or facilitate the implementation of a policy option [30] which in this case is the integration of mental health into PHC. Barriers will be defined as all factors that create obstacles or prevent progress towards attaining set goals whilst facilitators are all factors that make it easy or easier to attain a set goal or complete a set task. These "factors" may be physical or metaphorical. The content of the barriers and facilitators will be defined by the SURE guide. The "findings unit" shall either be predefined by the author or identified in a verbatim quote. In order to synthesize results across the included studies, a review of the reported outcomes for each study will be done [40] by EW and ZT to determine how the primary outcome of interest has been categorized along the SURE framework and reported for each study.

\section{Data synthesis}

Data synthesis will take on two synergistic approaches given that we plan to include both qualitative and quantitative studies. In the process of data extraction, key information will be categorized by study type and given codes which will be used in coding framework. The synthesis will adapt the "Best fit" framework synthesis by making reference to the extracted data from the included studies to construct a new evidence-based conceptual model regarding integration of mental health into primary health care [34]. The conceptual framework will be composed of a priori themes supported by evidence from the studies, plus new themes generated by the thematic analysis of evidence falling outside the framework. The conceptual framework will be assessed for bias and to determine if the synthesis is sensitive to the adjudged reported quality, design or location of included studies. Any differences between the a priori framework and the new framework will be explored when the synthesis is complete. This will test publication bias within the sample of included studies as well as the SURE framework for global use.

The process will be overseen by EO, a qualitative research methods expert on the review team, to ensure reflexivity, rigor, and quality.

\section{Discussion}

The SPIDER framework has been chosen for this review because of its suitable application to qualitative and mixed methods research and will be used as a guide when selecting articles for inclusion [33, 34]. We intend to leave our search open to any language with no date restriction and with broad search terms to capture potentially eligible studies that will ensure that our research question is answered. Data will be independently extracted to promote reflexivity during the process. Quality assessment tools will be applied to the selected articles to ensure quality of evidence presented.

Data extracted will be synthesized using the "Best fit" framework because it has been used before and proved its suitability in producing new conceptual models for explaining decision-making and possible behaviors [34]. In addition, evaluation of the review findings which will include attitudes and views among other unobservable and subjective constructs will enable us to understand the how and why of certain behaviors, decisions, and individual experience towards integration of mental health services into PHC. Synthesizing evidence from countries across the globe will provide useful insights into the experiences of integrating mental health services into $\mathrm{PHC}$ and how the barriers and challenges have been handled.

The findings will be used to explain reasons affecting implementation, predict how stakeholders respond, and/ or identify areas that are not functioning well within the health system [41]. They will be useful to a wide array of stakeholders involved in the implementation of the mental health integration into PHC. Examples of unsuccessful integration of mental health will not cause publication bias but simply present global experiences and best practices. 


\section{Additional files}

Additional file 1: PRISMA-P. (DOCX $31 \mathrm{~kb})$

Additional file 2: Search string. (DOCX $15 \mathrm{~kb}$ )

\section{Abbreviations}

CASP: Critical Appraisal Skills Program; PHC: Primary health care SURE: Supporting the Use of Research Evidence; WHO: World Health Organization

\section{Acknowledgements}

We would like to thank Professor Celestino Obua for supporting this review by providing training on how to conduct systematic reviews through his grant "Mbarara University Research Training Initiative (MURTI) Program (Grant Number D43TW010128) funded by the Fogarty International Center of the National Institutes of Health. The trainers came from the Africa Center for Systematic Reviews and Knowledge translation, College of Health Sciences, Makerere University, Kampala.

Appreciation also goes to Makerere-Sweden (Mak-Sida) Bilateral Program for supporting the review through facilitating EW (PhD student) to attend the "Information Competence and Management" training at Makerere University.

\section{Funding}

There is no dedicated funding; review is part of EW PhD work.

\section{Availability of data and materials}

Not applicable.

\section{Authors' contributions}

EW conceived the study and wrote the first draft with EO and ZT; and all authors revised the protocol. EW, DA, and AK developed the search strategy while the data abstraction form was developed by EW, with input from EO and ZT. CO is the senior researcher on the team providing overall guidance. RK and AM will do the data extraction. All authors read and approved the final manuscript.

\section{Ethics approval and consent to participate}

Not applicable.

\section{Consent for publication}

Not applicable.

\section{Competing interests}

The authors declare that they have no competing interests.

\section{Publisher's Note}

Springer Nature remains neutral with regard to jurisdictional claims in published maps and institutional affiliations.

\footnotetext{
Author details

${ }^{1}$ Office of Research Administration, Mbarara University of Science and Technology, Mbarara, Uganda. ${ }^{2}$ Department of Psychiatry, College of Health Sciences, Makerere University, Kampala, Uganda. ${ }^{3}$ Africa Center for Systematic Reviews and Knowledge Translation, College of Health Sciences, Makerere University, Kampala, Uganda. ${ }^{4}$ Library, Africa Center for Systematic Reviews and Knowledge Translation, College of Health Sciences, Makerere University, Kampala, Uganda. ${ }^{5}$ Department of Anatomy, Mbarara University of Science and Technology, Mbarara, Uganda. ${ }^{6}$ Department of Pharmacology and Therapeutics and Vice Chancellor, Mbarara University of Science and Technology, Mbarara, Uganda. ${ }^{7}$ Department of Medicine \& of Health Policy, George Washington University, Washington DC, USA. ${ }^{8}$ Mbarara University of Science and Technology, Mbarara, Uganda.
}

Received: 7 March 2017 Accepted: 9 August 2017

Published online: 25 August 2017

\section{References}

1. World Health Organization. Mental health: a state of well-being 2014 [updated August 2014. Available from: http://www.who.int/features/factfiles/ mental_health/en/.

2. World Health Organization. Comprehensive mental health action plan 2013-2020. Geneva: World Health Organization; 2013.

3. Saraceno B, van Ommeren M, Batniji R, Cohen A, Gureje O, Mahoney J, et al. Barriers to improvement of mental health services in low-income and middle-income countries. Lancet. 2007;370(9593):1164-74.

4. World Health Organization. Mental health policy, planning \& service develop integrating systems \& services, integrating people. Geneva: World Health Organization; 2007.

5. Charlson FJ, Diminic S, Lund C, Degenhardt L, Whiteford HA. Mental and substance use disorders in sub-Saharan Africa: predictions of epidemiological changes and mental health workforce requirements for the next 40 years. PLoS One. 2014;9(10):e110208.

6. Whiteford HA, Ferrari AJ, Degenhardt L, Feigin V, Vos T. Global Burden of Mental, Neurological, and Substance Use Disorders: An Analysis from the Global Burden of Disease Study 2010. In: Patel V, Chisholm D, Dua T, Laxminarayan R, MedinaMora ME, editors. Mental, Neurological, and Substance Use Disorders: Disease Control Priorities, Third Edition (Volume 4). Washington (DC): The International Bank for Reconstruction and Development/The World Bank. (c) 2016 International Bank for Reconstruction and Development/The World Bank: 2016.

7. World Health Organization. The world health report 2008: primary health care now more than ever. 2008.

8. Whiteford HA, Degenhardt L, Rehm J, Baxter AJ, Ferrari AJ, Erskine HE, et al. Global burden of disease attributable to mental and substance use disorders: findings from the Global Burden of Disease Study 2010. Lancet (London, England). 2013;382(9904):1575-86.

9. World Health Organization, World Organization of National Colleges Academies, Academic Associations of General Practitioners/Family Physicians. Integrating mental health into primary care: a global perspective. In: Organization WH, editor. Geneva: WHO Press; 2008.

10. World Health Organization. Declaration of alma-ata: World Health Organization Regional Office for Europe; 2004

11. World Health Organization, Unicef. Primary health care: a joint report. 1978.

12. TO A. Health for all beyond 2000: the demise of the Alma-Ata Declaration and primary health care in developing countries. Med J Aust. 2003:178(1):17-20.

13. Hall JJ, Taylor R. Health for all beyond 2000: the demise of the Alma-Ata Declaration and primary health care in developing countries. Med J Aust. 2003;178(1):17-20.

14. World Health Organization. Improving health systems and services for mental health. 2009.

15. McGough PM, Bauer AM, Collins L, Dugdale DC. Integrating behavioral health into primary care. Popul Health Manag. 2016;19(2):81-7.

16. Kakuma R, Minas H, van Ginneken N, Dal Poz MR, Desiraju K, Morris JE, et al Human resources for mental health care: current situation and strategies for action. Lancet. 2011;378(9803):1654-63.

17. Force CPST. Recommendation from the community preventive services task force for use of collaborative care for the management of depressive disorders. Am J Prev Med. 2012:42(5):521-4.

18. Davis K. Costs and consequences of enhanced primary care for depression: systematic review of randomised economic evaluations. Primary Health Care. 2007;17(1):34.

19. Petersen I, Marais D, Abdulmalik J, Ahuja S, Alem A, Chisholm D, et al. Strengthening mental health system governance in six low-and middleincome countries in Africa and South Asia: challenges, needs and potential strategies. Health Policy Plan. 2017;32(5):699-709.

20. Sheikh K, Gilson L, Agyepong IA, Hanson K, Ssengooba F, Bennett S. Building the field of health policy and systems research: framing the questions. PLoS Med. 2011;8(8):e1001073.

21. Barraclough F, Longman J, Barclay L. Integration in a nurse practitioner-led mental health service in rural Australia. Aust J Rural Health. 2016;24(2):144-50.

22. Bindman J, Johnson S, Wright S, Szmukler G, Bebbington P, Kuipers E, et al. Integration between primary and secondary services in the care of the severely mentally ill: patients' and general practitioners' views. Br J. Psychiatry. 1997;171:169-74. 
23. Chowdhury N. Integration between mental health-care providers and traditional spiritual healers: contextualising islam in the twenty-first century. J Relig Health. 2016;55(5):1665-71.

24. Bhana A, Petersen I, Baillie KL, Flisher AJ. Implementing the World Health Report 2001 recommendations for integrating mental health into primary health care: a situation analysis of three African countries: Ghana, South Africa and Uganda. Int Rev Psychiatry. 2010;22(6):599-610.

25. Bhana A, Petersen I, Baillie KL, Flisher AJ. Implementing the World Health Report 2001 recommendations for integrating mental health into primary health care: a situation analysis of three African countries: Ghana, South Africa and Uganda. Int Rev Psychiatry (Abingdon, England). 2010;22(6):599-610

26. Abera M, Tesfaye M, Belachew T, Hanlon C. Perceived challenges and opportunities arising from integration of mental health into primary care: a cross-sectional survey of primary health care workers in south-west Ethiopia. BMC Health Serv Res. 2014;14:113.

27. Ackerman B, Pyne JM, Fortney JC. Challenges associated with being an offsite depression care manager. J Psychosoc Nurs Ment Health Serv. 2009; 47(4):43-9.

28. Society the Individual and Medicine. Primary Care: Definitions and Historical Developments Canada: University of Ottawa; 2016 [updated February 10, 2017. Available from: https://www.med.uottawa.ca/sim/data/Primary_Care. htm.

29. Glenton C, Colvin CJ, Carlsen B, Swartz A, Lewin S, Noyes J, et al. Barriers and facilitators to the implementation of lay health worker programmes to improve access to maternal and child health: qualitative evidence synthesis. Cochrane Database Syst Rev. 2013(10)

30. The SURE Collaboration. SURE guides for preparing and using evidencebased policy briefs: identifying and addressing barriers to implementing policy options. Version 2.1 [updated November 2011]. The SURE Collaboration; 2011. Available from www.evipnet.org/sure.

31. Shamseer L, Moher D, Clarke M, Ghersi D, Liberati A, Petticrew M, et al. Preferred reporting items for systematic review and meta-analysis protocols (PRISMA-P) 2015: elaboration and explanation. BMJ. 2015;349:97647.

32. Shamseer L, Moher D, Clarke M, Ghersi D, Liberati A, Petticrew M, et al. Preferred reporting items for systematic review and meta-analysis protocols (PRISMA-P) 2015: elaboration and explanation. BMJ (Clinical research ed). 2015;349:97647.

33. Cooke A, Smith D, Booth A. Beyond PICO: the SPIDER tool for qualitative evidence synthesis. Qual Health Res. 2012;22(10):1435-43.

34. Carroll C, Booth A, Leaviss J, Rick J. "Best fit" framework synthesis: refining the method. BMC medical research methodology. 2013;13(1):37.

35. Verboom B, Montgomery P, Bennett $S$. What factors affect evidenceinformed policymaking in public health? Protocol for a systematic review of qualitative evidence using thematic synthesis. Syst Rev. 2016;5(1):61.

36. Noyes J, Popay J. Directly observed therapy and tuberculosis: how can a systematic review of qualitative research contribute to improving services? A qualitative meta-synthesis. J Adv Nurs. 2007;57(3):227-43.

37. Carroll C, Booth A, Lloyd-Jones M. Should we exclude inadequately reported studies from qualitative systematic reviews? An evaluation of sensitivity analyses in two case study reviews. Qual Health Res. 2012;22(10): $1425-34$.

38. Thomas H. Quality assessment tool for quantitative studies. Toronto: McMaster University; 2003.

39. Thomas H. Quality assessment tool for quantitative studies. Toronto: Effective Public Health Practice Project McMaster University; 2003.

40. Kay-Lambkin FJ, Thornton L, Lappin JM, Hanstock T, Sylvia L, Jacka F, et al. Study protocol for a systematic review of evidence for lifestyle interventions targeting smoking, sleep, alcohol/other drug use, physical activity, and healthy diet in people with bipolar disorder. Syst Rev. 2016;5(1):106.

41. Gale NK, Heath G, Cameron E, Rashid S, Redwood S. Using the framework method for the analysis of qualitative data in multi-disciplinary health research. BMC Med Res Methodol. 2013;13(1):117.

\section{Submit your next manuscript to BioMed Central and we will help you at every step:}

- We accept pre-submission inquiries

- Our selector tool helps you to find the most relevant journal

- We provide round the clock customer support

- Convenient online submission

- Thorough peer review

- Inclusion in PubMed and all major indexing services

- Maximum visibility for your research

Submit your manuscript at www.biomedcentral.com/submit
Biomed Central 